\title{
Proposta de um Framework para aplicações web
}

\section{Proposal for a Framework for web applications}

\author{
Francisco de Oliveira Dantas Filho ${ }^{1}$ \\ Hevanderson da Silva ${ }^{1}$ \\ Angelo Márcio de Paula ${ }^{2}$
}

Palavras-chaves:

Web

Software

Padrões de Projeto

Arquitetura

\section{Resumo}

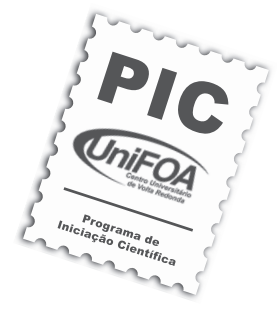

Artigo

Original

Original

Paper

Aplicações envolvendo internet são denominadas aplicações web e requerem requisitos especiais de arquitetura de software para um correto desenvolvimento e funcionamento. Este trabalho tem como proposta apresentar as técnicas de software livre, formando uma arquitetura para construir aplicações web que funcionem na internet ou intranet. Todo framework foi desenvolvido na plataforma Java com o uso de Servlet, JSP e JavaBean. Ao final, o processo realizado apresentou alta produtividade, pois retirou do desenvolvedor a responsabilidade de programar o código de comunicação envolvendo o protocolo HTTP e as trocas de mensagens entre as camadas da aplicação. Desta forma, o programador se concentrará em programar somente a regra de negócio do sistema proposto.

\begin{abstract}
Applications involving Internet are called web applications and require specific requirements for software architecture for a correct development and functioning. This work has a proposal present the techniques of free software forming architecture to build web applications operating on the internet or intranet. Finally, the processor conducted showed high productivity, because withdrew from the developer the responsibility to set the code of communication involving the HTTP protocol and the exchange of messages between application tiers. Thus, the developer will focus on programming only the business rule of the proposed system.
\end{abstract}

Key words:

Web

Software

Design Pattern

Architecture

\section{Introdução}

Aplicações de software são complexas porque modelam a complexidade do mundo real. Atualmente, aplicações típicas são muito grandes e complexas para que um único indivíduo consiga compreendê-la. Sobre essas circunstâncias, a construção e a manutenção de grandes aplicações tornam-se um sério problema.

A demanda por mais serviços, novos produtos, pela melhoria da qualidade e menores preços vêm, já há um bom tempo, impulsio- nando o estudo de técnicas de desenvolvimento de software que possam ser efetivamente utilizadas para a obtenção desses objetivos. Além do aspecto puramente técnico, encontramos ainda enorme dificuldade em gerenciar o processo de desenvolvimento.

Este trabalho reúne as técnicas denominadas Padrões de Projetos para construção de aplicações que usam a internet/intranet como meio de comunicação, formando um framework, que atinja desde o cliente, executando uma página no navegador, até o dado, 
sendo gravado no banco de dados, ou seja, uma proposta de uma arquitetura para desenvolvimento web em uma totalidade razoável.

Todos os testes foram realizados com produtos open source, destacando-se a linguagem de programação Java através das tecnologias Servlet e JSP que são especialidades para desenvolvimento web.

Ao final, é apresentado um framework com uma arquitetura madura, pronta para ser usada na construção de aplicações web por qualquer equipe de desenvolvimento, a qual tenha conhecimentos básicos na linguagem Java.

\section{Revisão Bibliográfica}

\subsection{Trabalhos Realizados}

Entre os trabalhos pesquisados no meio acadêmico e na comunidade de desenvolvedores de código aberto (open source), destacamse sob o ponto de vista acadêmico e contribuíram para o desenvolvimento desta pesquisa:

- Framework Apache Struts, desenvolvido por Struts (2009);

- Framework JavaServer Faces, desenvolvido por SDN (2009).

O Apache Struts é um projeto popular desenvolvido por The Apache Software Foundation (STRUTS, 2009). Trata-se de um projeto open source para desenvolvimento de aplicações web usando a linguagem de programação Java. O Struts é empregado onde há necessidade de criação de web sites que possuem respostas dinâmicas. Aplicações web baseadas em JSP (Java Server Pages), ASP (Active Server Pages) e PHP (Hypertext Preprocessor) misturam dados, controle e visualização em apenas um componente e, na prática, verifica-se uma dificuldade na manutenção destes sites, principalmente, quando se trata de sistemas de médio para grande porte. Então, umas das preocuções em uma aplicação é ter uma clara separação das camadas, o que é alcançado com a utilização da arquiteura MVC (Model-View-Controller). O Struts foi concebido para ajudar os desenvolvedores criar aplicações web que utilizam a arquitetura
MVC. Esse projeto é mantido por um grupo voluntariado que colabora entre si desenvolvendo o framework e mantendo-o dentro das características de código aberto.

O framework JavaServer Faces também é usado para desenvolvimento de aplicações web dinâmicas visando à separação das camadas. É mantido pelo Java Community Process (JSR-314) e estabelece o padrão do lado servidor para a construção de interfaces com o usuário. Com as contribuições do grupo de especialistas, o JSF foi concebido de forma a ser aproveitado por ferramentas as quais irão tornar ainda mais fácil o desenvolvimento de aplicações web.

A Facilidade de uso é a principal meta do JSF, pois ele define claramente uma separação entre a aplicação lógica e a camada de apresentação tornando fácil a ligação entre a camada de apresentação e a lógica do negócio. Esse projeto permite, a cada membro da equipe de desenvolvimento, concentrar-se no desenvolvimento da lógica de negócio, ficando a cargo do JSF as complexidades de ligação entre as camadas. Por exemplo, o desenvolvedor não precisa ser especialista em HTML, pois o JSF possui TAG's que encapsulam a regra de apresentação através de passagem de parâmetros, sem a necessidade de escrever qualquer script (SDN ,2009).

\subsection{Revisão Tecnológica}

\subsubsection{Java Beans}

JavaBeans são componentes de software escritos em Java. A API de JavaBeans foi criada pela Sun Microsystem com a cooperação da indústria e determina as regras que o programador de software deve seguir a fim de criar componentes de software reutilizáveis e independentes. Assim como muitos outros componentes de software, os Beans encapsulam tanto estado quanto comportamento. Ao usar a coleção de tags relacionadas a Beans de JSP nas suas páginas da web, os programadores de conteúdo podem alavancar o poder de Java para adcionar elementos dinâmicos às suas páginas sem escrever uma única linha de código Java.

Então, um JavaBean é uma classe que segue certo padrão de projeto. O padrão tem 
uma semântica bem definida para permitir que a estrutura do JavaBean seja descoberta através da introspecção de sua classe compilada, usando a API de reflexão Java. Os requisitos fundamentais de um JavaBean são os seguintes: Ser uma classe Java public, possuir um construtor sem nenhum argumento e, normalmente, possuir uma ou mais propriedades (ou atributos) que podem ser manipulados através de métodos públicos (DEITEL E DEITEL, 2006).

\subsubsection{Servlet}

Um Servlet Java é um programa no lado do servidor que é ativado pelo servidor web para atender aos pedidos HTTP. Um Servlet é executado em uma máquina virtual Java no servidor da web e, normalmente, realiza alguma computação para gerar o conteúdo da resposta HTTP (DEITEL E DEITEL, 2006). Em comparação a outras técnicas de programação no lado do servidor, os servlets representam uma alternativa mais madura e confiável.

\subsubsection{JSP}

Devido ao grande sucesso que é o ASP (Active Server Page) da Microsoft, a Sun Microsystem decidiu construir uma versão tão poderosa quanto ao ASP da Microsoft, o JavaServer Pages (JSP). A tecnologia JSP é incorporado ao modelo de Servlet. A ideia básica das JSP é permitir que o código Java seja misturado com modelos HTML e XML estáticos. O propósito da lógica Java é gerar conteúdo dinâmico na página, enquanto a linguagem de marcação manipula a estruturação e a apresentação dos dados. (DEITEL E DEITEL, 2006).

\section{Materiais e Métodos}

\subsection{Arquitetura Aplicação}

Os dois frameworks (Struts e JSF) dominam o mercado de desenvolvimento web, porém, eles apenas tratam a camada que faz a interface com os pedidos HTTP. Além do mais, como eles cobrem praticamente todos os pontos relacionados ao desenvolvimento web, os mesmos se tornam complexos, exigindo um conhecimento extra além dos JSP, Servlets e JavaBean.
A proposta desta pesquisa é apresentar um framework para desenvolvimento web extendido até a camada da regra de negócio e a camada de dados sem ter que apresentar conhecimentos além do trivial (JSP, Servlet e JavaBean).

A arquitetura da aplicação deve ser projetada de modo que determinadas situações típicas não se tornem problemas os quais impedirão a aplicação de atender aos requisitos do sistema. São consideradas as seguintes situações como críticas:

- Sistemas envolvendo interfaces, servidor de aplicação remoto e banco de dados.

- Aplicações distribuídas.

- Aplicações que acessam dados em mais de uma fonte de dados.

\subsubsection{Sistemas envolvendo interfaces, servi- dor de aplicação remoto e banco de dados}

As seguintes situações estão presentes nesse caso:

- O sistema tem que aceitar dados do usuário, atualizar o banco de dados e retornar, mais tarde e em outro cenário, o dado para o usuário.

- A existência de várias formas em que o dado pode ser aceito e apresentado no sistema do usuário.

- Dados que alimentam um sistema em um formulário deverão ser restauráveis em outro formulário.

Se o sistema desenvolve um simples componente que interage com o usuário e também mantém o banco de dados, então um requerimento para suportar um novo tipo de apresentação necessitará o reprojeto do componente.

No estudo de caso proposto, a clínica fornece a facilidade de acompanhamento dos resultados. Quando o usuário se autenticar no sistema, se ele for o paciente, ele verificará o resultado de um determinado procedimento; se ele for o administrador, verificará a quantidade de procedimentos realizados em um

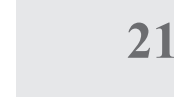

\section{(1)}

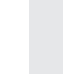

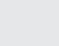

\section{(1)}

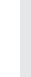

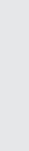


período de tempo; se ele for o fisioterapeuta, dará um parecer no procedimento realizado. Ou seja, a mesma informação pode ser visualizada de várias formas, de acordo com o perfil do solicitante. Aqui, o mesmo dado que representa o procedimento é visto em múltiplas formas, mas é controlado por uma única entidade na aplicação. Assim, três tarefas ocorrem ao mesmo tempo na aplicação:

- Gerenciar a interação do usuário com o sistema.

- Gerenciar o dado atual.

- Formatar o dado em múltiplas formas.

Dessa forma, um simples componente que faz todas as tarefas pode ser dividido dentro de três componentes independentes. Todas três tarefas podem ser tratadas por diferentes componentes. Então, a solução segundo Deshmukh e Malavia (2003) é separar os dados da apresentação dos dados de manutenção e ter um terceiro componente coordenador. Esses três componentes são chamados "Modelo", "Visão" e "Controlador". Eles formam o básico do padrão MVC. A Figura 1 mostra o relacionamento entre os componentes do modelo MVC.

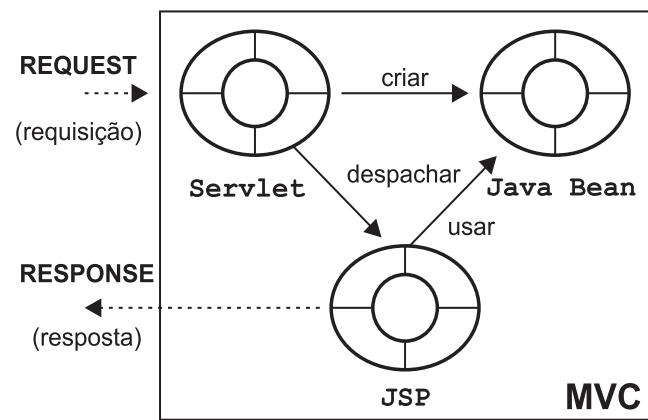

Figura 1. Modelo-Visão-Controlador

O modelo é responsável por manter o dado ou o estado da aplicação. Ele também gerencia o armazenamento e recuperação do dado armazenado. O componente JavaBean em conjunto com o framework Hibernate que realiza persistência objeto relacional foi aplicado nessa camada.

A visão contém a lógica de apresentação. Ela mostra o dado contido no modelo para o usuário. Ele também permite o usuário interagir com o sistema e notificar o controla- dor às ações do usuário. Os componentes web JavaServer Page (JSP) e o JSP Standard Tag Library (JSTL) foram aplicados na construção dessa camada.

O Controlador gerencia o modelo e a visão. Ele instancia e associa o modelo e a visão. Dependendo do requerimento da aplicação, ele pode instanciar múltiplas visões e pode associá-las com o mesmo modelo. Ele escuta as ações do usuário e manipula o modelo de acordo com a regra de negócio. Um Servlet foi aplicado nessa camada.

Desenvolver uma aplicação seguindo o padrão model-view-controller permite criar múltiplas visões para um mesmo dado. Mudanças ocorrem nos componentes do modelo ou nos componentes de visão de forma independente. A visão permanece a mesma. Isso aumenta a manutenibilidade e a extensabilidade do sistema.

\subsubsection{Em aplicações distribuídas}

Os componentes do cliente e os componentes do servidor residem em locais remotos e a comunicação ocorre sobre a rede de comunicação. Sendo assim:

- O banco de dados fica no lado do servidor

- O servidor fornece métodos get's (getImagem( ), por exemplo) para que o mesmo possa chamá-lo um por um para reaver os dados.

- O servidor fornece métodos set's (setImagem(byte image[]), por exemplo, para o cliente, para que o mesmo possa chamá-lo um por um para atualizar os dados no banco de dados.

Cada chamada entre o cliente e o servidor é uma chamada de métodos remotos com uma substancial sobrecarga na rede. Se a aplicação cliente chama os métodos get's e set's para reaver ou atualizar simples valores de atributos, ocorrerão muitas chamadas remotas para esse atributo. Essa chamada remota gera muita sobrecarga na rede e, consequentemente, uma diminuição do desempenho do sistema.

A camada de negócio acessará o banco de dados diretamente ou via a camada de recursos, e colocará o mecanismo de acesso dentro de um conjunto de entidades e beans 
controladores. Esses componentes expõem o dado via interfaces remotas. As aplicações desktop `s, os servlets e páginas JSP na camada de apresentação precisam acessar esses dados de negócio, eles fazem isso chamando os métodos das interfaces remotas implementadas por Javabeans.

Para exemplificar, vamos considerar que desejamos incluir dados de uma paciente na base de dados e se trata de uma aplicação distribuída. Os dados são: identificador, nome e imagem (foto), o qual está encapsulado em um Javabean chamado Laudo. O acesso para a informação está encapsulado pela aplicação da camada de negócio com a ajuda de um Javabean controlador chamado LaudoFacade. Esse controlador expõe métodos para o cliente remoto, tais como: getId(), setId(...), getNome(), setNome(...), getImagem(), setImagem(...). Os servlets e páginas JSP então têm que chamar cada método um por um remotamente no servidor. Conforme a Figura 2.

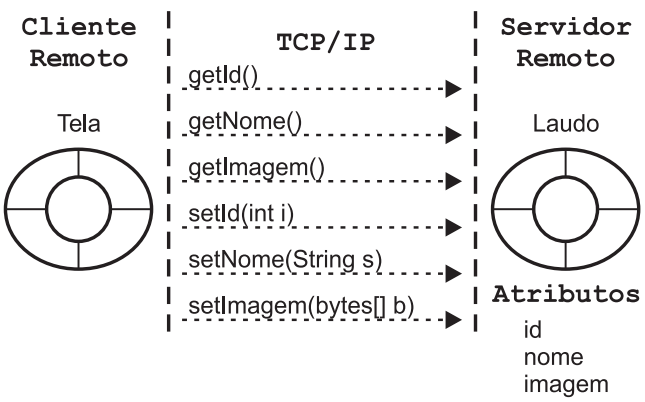

Figura 2 - Sobrecarga de chamadas em objetos remotos

A Figura 2 apresenta alguns problemas:

- Um simples objeto de negócio tem muitos atributos

- $\quad \mathrm{Na}$ maioria das vezes, o cliente requer vários atributos ao mesmo tempo e quase nunca apenas um atributo.

- A utilização dos métodos get's é muito maior que os métodos set's

Dessa forma, é preciso criar um objeto para encapsular todos os atributos que são requeridos pela aplicação cliente. Esse objeto é chamado de Value Object. Quando o cliente requer o dado do servidor, o componente do lado do servidor extrai os valores localmente e constrói o Value Object. Esse Value Object é então enviado para o cliente por valor e não por referência, o que significa que esse objeto é serializado e transferido pela rede bit a bit. $\mathrm{O}$ cliente do outro lado reconstrói o objeto localmente com todos os valores intactos. Uma vez que o objeto está local, o cliente consulta todos os atributos sem gerar sobrecarga na rede.

Agora, considerando o exemplo anterior, ao invés de fazer múltiplas chamadas remotas no objeto Laudo para reaver todos os atributos para formar um laudo, o cliente chama um simples método getLaudo no LaudoFacade, este vai extrair os dados do objeto Laudo, criar o LaudoVO e retorná-lo pela rede com todos os atributos estruturados em um objeto próprio para trafegar pela rede. Assim, o cliente acessará todas as informações em uma única vez localmente. A Figura 3 está ilustrando a solução.

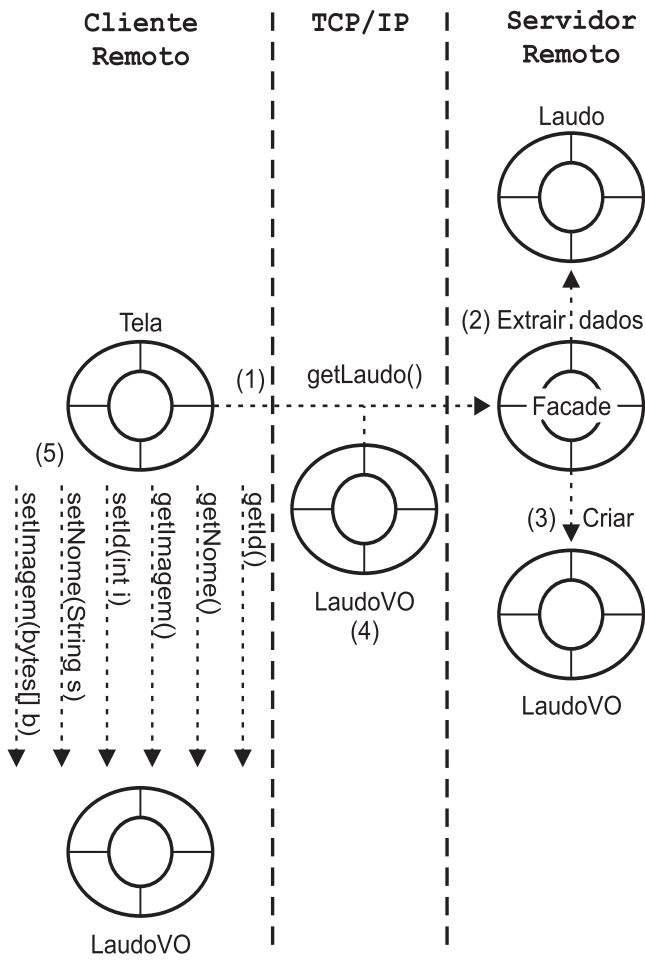

Figura 3 - Aplicação dos padrões Facade e Value Object

O cliente pode ser uma página web JSP, um servlet, uma applet Java, um JPanel no formato desktop que faz chamadas remotas para o objeto de negócio na camada de negócio no servidor. O objeto de negócio é um componente que cria o Value Objetct. O cliente sempre fará chamadas para o objeto de negócios.

A interface remota é simplificada porque múltiplos métodos retornando um simples valor são simplificados dentro de um simples método, retornando um grupo de múltiplos valores. A redução do número de chamadas, por meio da rede, melhora o tempo de resposta da 
aplicação. Se o cliente deseja atualizar o valor do atributo, ele primeiro atualiza o valor no Value Object local e, então, o envia para o servidor para que possa ser persistido o novo valor. Tudo isso acontece usando o mecanismo de passagem por valor.

O Value Object pode ficar desatualizado, isto é, se o cliente adquiriu um Value Object por um longo período de tempo, há possibilidade de a informação ter sido atualizada por outro cliente. No caso de Value Object mutável, requisições de atualização de dois ou mais clientes podem resultar em conflito de dados.

Resumindo, um Value Object é um pequeno objeto Java serializado que é usado para conduzir grupo de dados sobre a rede de um componente residente em outra camada de uma aplicação multicamadas distribuídas. $\mathrm{O}$ seu principal propósito é reduzir sobrecarga de comunicação, reduzindo o número de chamadas remotas entre os componentes distribuídos.

\subsubsection{Em aplicações que acessam dados em mais de uma fonte de dados}

As aplicações envolvem atualizações e consultas de fontes de dados diferentes: banco de dados relacionais, sistemas de arquivos, arquivos $X M L$, assim por diante. As seguintes situações ocorrem:

- As aplicações envolvem atualizações e consultas de fontes de dados iguais, como um banco de dados, mas bancos de dados são fornecidos por diferentes fabricantes, por exemplo, Oracle, SQLServer da Microsoft, DB2 da IBM e outros, onde cada fabricante construiu o seu próprio mecanismo de acesso.

- Alguns bancos de dados permitem manipulação de dados via store procedure. $\mathrm{O}$ processo de chamada da store procedure são dependentes da implementação do sistema do banco de dados.

A Figura 4 mostra a dificuldade que a camada de negócio encontra para acessar dados de diferentes fontes, visto que cada fonte de dados possui o seu próprio mecanismo de acesso. Outro problema que existe também é quando a camada de negócio precisa acessar apenas uma fonte de dados, mas o banco de dados precisa mudar de endereço físico em algum momento, logo, todos os componentes da camada de negócio que acessam a fonte de dados diretamente serão alterados. Então, quando temos várias fontes de dados ou quando a mesma não está corretamente definida, apresentará um grande problema de manutenção e de baixa flexibilidade do sistema.

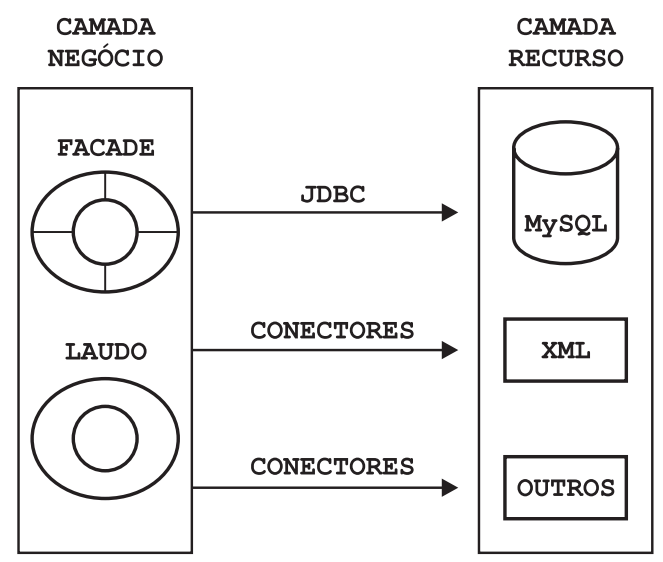

Figura 4 - Negócio acessando base de dados

Dessa forma, a lógica de negócio implementada pelos objetos de negócio não deve depender do tipo do armazenador de dados que é usado. $\mathrm{O}$ algoritmo de negócio deve ser separado do código que acessa o banco de dados. A lógica de negócio implementada pelo objeto de negócio não deverá depender da forma em que o dado armazenado é acessado.

A solução apresentada na Figura 5 cria um objeto especial que se ocupa em acessar os bancos de dados. O DAO é uma classe abstrata ou uma interface que tem métodos como select(..), upadate(..), delete(...) e insert(..), as classes derivadas deverão então implementar esses métodos na forma específica que é requerida pelo banco de dados individualmente. Então os Dao's encapsulam a lógica de acesso aos dados, e o objeto de negócio que usa as classes Dao's não precisa conhecer sobre algum conjunto de código $S Q L$ ou as API's de baixo nível fornecidas pelo fabricante do banco de dados.

O padrão $D A O$ fornece uma camada de abstração entre as camadas de negócio e a fonte de dados. Objetos da camada de negócio acessam a fonte de dados via Data Access Object. O DAO encapsula os detalhes de persistência e fornece um conjunto padrão de interface para acessar o dado. Esse objeto realiza todas as ope- 
rações relacionadas à fonte de dados, tais como, consultas SQL. Isso limita o impacto de mudanças da fonte de dados para apenas as classes que compõe o $D A O$, isso melhora a flexibilidade e manutenibilidade dos objetos de negócio.

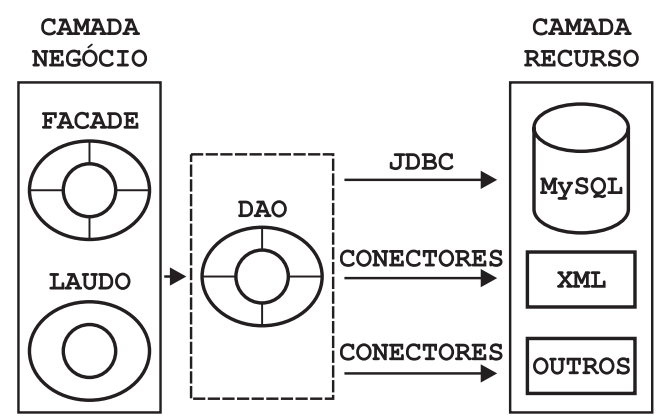

Figura 5 - Exemplo do padrão DAO

O objeto de negócio usa o $D A O$ para alcançar o dado armazenado, o próprio $D A O$ abstrai e encapsula as características da fonte de dados que a camada de negócio precisa para acessar os dados. Dessa forma, a fonte de dados será qualquer espécie de banco de dados ou sistema legado, e a aplicação vai selecionar dinamicamente a fonte de dados plugando o apropriado $D A O$.

\subsection{Arquitetura da Aplicação proposta}

A aplicação será construída com componentes para as camadas cliente, apresentação, negócio, integração e dados e foi influenciada por Alur, Crupi e Malks (2001), Deshmukh e Malavia (2003) e Paula (2007). A Figura 6 mostra os padrões cooperando entre si, formando a arquitetura.

A camada do cliente permitirá ao cliente interagir com a aplicação, essa pode ser uma aplicação web rodando em um browser, uma aplicação desktop ou até uma aplicação Wirelles rodando em um Palm. A camada de apresentação define o nível de permissão do usuário, encapsula a lógica da interface, gerencia a sessão do usuário e recebe e envia respostas para o usuário. A camada de negócios é o módulo responsável pela realização das regras de negócio da aplicação. A camada de integração é responsável pela comunicação com os recursos externos. A camada de recursos é responsável por manter os dados persistidos.

Dessa forma, o cliente web fará sempre uma requisição para um Front Controller, que delegará a um Dispatcher a função de criar um action para atender a solicitação e encaminhar a resposta para um View formar a saída para o cliente, essa funcionalidade também é mostrada na Figura 7. O cliente Desktop acessará o Facade diretamente através de uma chamada remota. O padrão Service Locator, faz a localização dos objetos remotos.

O Facade é um controlador da regra de negócio. Ele conhece o fluxo de atendimento da aplicação, sua principal função é acessar a base de dados através dos Dao `s criados pelos Abstracts Factory e Factory, executar a regra de negócio encapsulada pelos objetos de negócios e garantir a resposta para o cliente criando Value Objets apropriados para cada solicitação. O sistema possuirá Implementation Dao para cada meio de armazenamento no sistema. Os meios mais comuns são XML, Sistema de arquivo e banco de dados relacional.

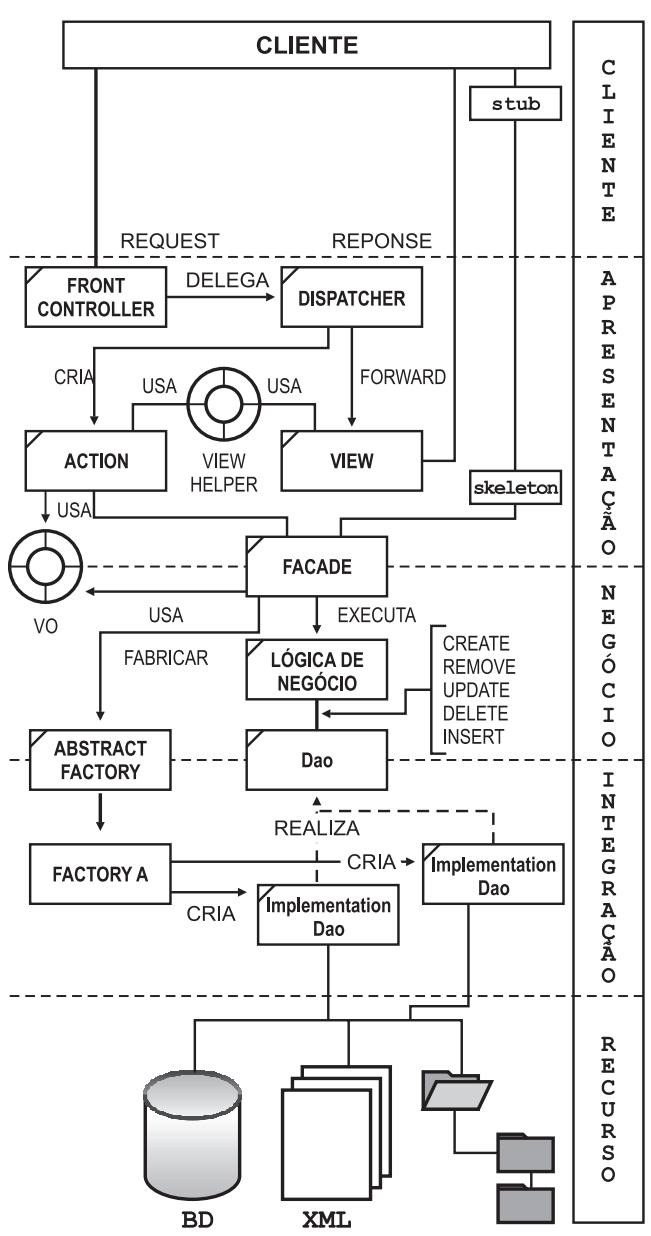

Figura 6-Arquitetura da aplicação segundo Paula (2007)

\section{Resultados}

A Figura 7 mostra as classes envolvidas no controlador da camada de apresentação. As classes são empacotadas em um arquivo com extensão jar, nesse caso, a sua reutilização é 
feita adicionando o arquivo de nome controle. jar no path da aplicação e a devida configuração do web.xml.

Então, quando o cliente disparar uma URL (Exemplo: pesquisar.do) o servlet FrontController será acionado pelo servido web e várias ações ocorreram no servidor:

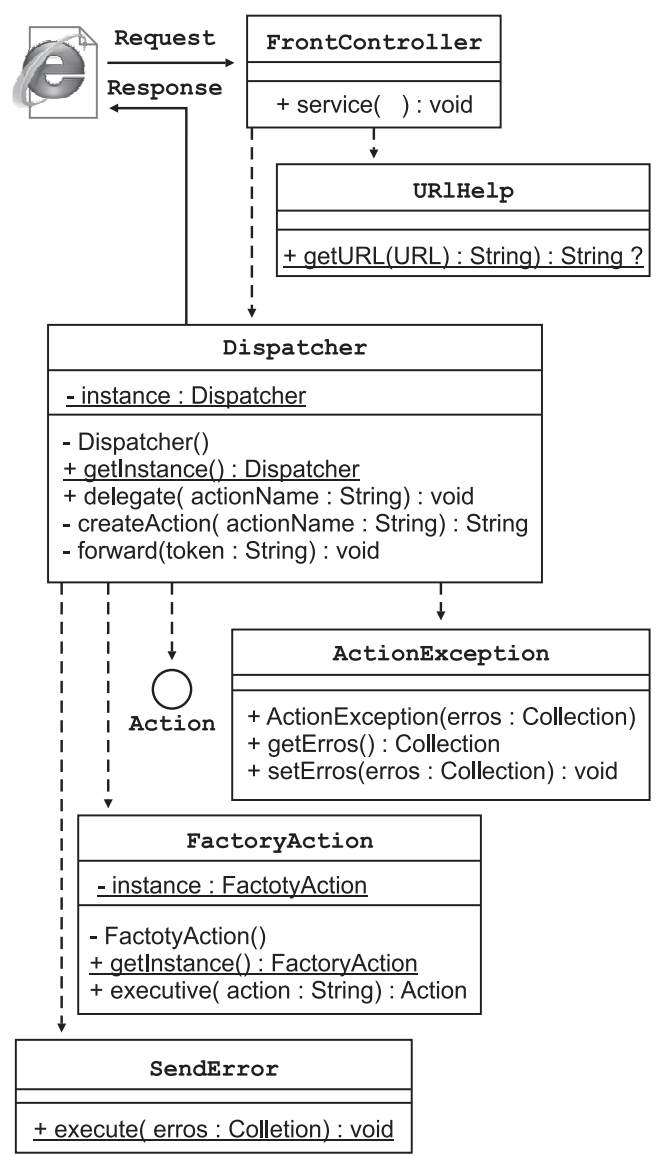

Figura 7- Controlador da camada de apresentação

1. Uma Thread FrontController é disparado.

2. A objeto URIHelp reconhece a URI solicitante.

3. O FrontController solicita uma instância do objeto Dispatcher.

4. O FrontController delega as demais responsabilidades para o objeto Dispatcher.

5. O Dispatcher solicita uma instância do objeto FactoryAction.

6. O Dispatcher pede ao FactoryAction o action presente na URI.

7. O Dispatcher executa o objeto Action (Exemplo: pesquisarAction.class)

8. O Dispatcher faz um forward (encaminha o pedido) para o documento (JSP, HTML, etc..) conforme o retorno do Action.
A Figura 8 evidencia o protótipo construído para testar o framework.

\section{Sistema de Controle da Clínica de Fisioterapia \\ Centro Universitário Oswaldo Aranha \\ Home Paciente Professor Estagiário Anamnese}

Consultar Paciente

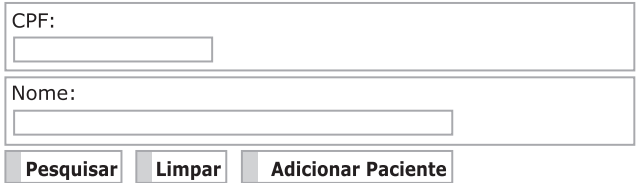

Figura 8- Estudo de caso

\section{Discussão}

Temos, ao longo dos anos, vários casos que indicam insucesso quando se trata de desenvolvimento de software. De forma empírica, constatamos as seguintes situações relatadas na Figura 9. Temos que unir esforços para atingirmos o topo da pirâmide e com um custo, qualidade e prazo dentro da normalidade.

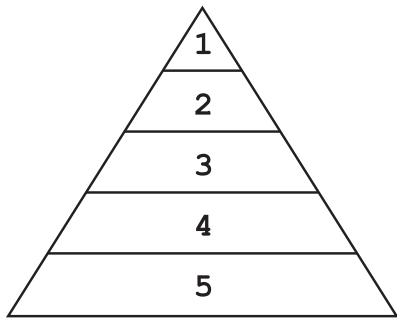

\footnotetext{
1. Produção com sucesso

2. Retrabalho

3. Grande retrabalho

4. Entregue, mas usuário não usa

5. Pagos, mas não entregues
}

Figura 9- Crise do software

Vários fatores influenciam no desenvolvimento do software e destacamos os mais importantes: alterações nas metas, falha no gerenciamento de riscos, complexidade do software e projeto da arquitetura. A nossa pesquisa é aplicada ao Projeto da Arquitetura e pretende-se, dessa forma, uma arquitetura que permita atender aos princípios básicos de uma aplicação: Performance; transparência; flexibilidade; confiabilidade e escalabilidade. Todos os membros da equipe precisam codificar da mesma forma, facilitando a rotatividade dos profissionais, fato concreto na atual escassez de profissionais de TI. Com isso, a nossa proposta contribuirá tanto no desenvolvimento devido à padronização imposta na manutenção 
do sistema devido ao uso dos padrões de projeto- quanto na sedimentação do sistema, pois está construído com boas regras de codificação, conhecidas e usadas mundialmente.

\section{Conclusão}

Concluímos que, devido à importância alcançada pelas aplicações web e a forma que a internet invadiu o nosso cotidiano, seja necessário realizar pesquisa para auxiliar na construção dos softwares, através de soluções reutilizáveis.

Percebemos também que no decorrer do desenvolvimento de uma aplicação, é comum nos depararmos com problemas que precisam de conhecimentos estratégicos para serem resolvidos. Para cada problema que encontramos, imediatamente, é considerado inúmeras formas de resolvê-lo, incluindo soluções de sucesso já vividas ou soluções completamente novas. O segredo é documentar cada solução aplicada e à medida que reusamos uma solução com sucesso, considera-se que temos uma solução para resolver um determinado problema.

Dessa forma esse framework apresentado poderá ser evoluído para atender a novas especificações, desde que devidamente documentado e compartilhado na comunidade acadêmica de desenvolvedores.

Finalizando, esse framework está maduro para ser usado em desenvolvimento de aplicações web, pois foi desenvolvido com técnicas já testadas mundialmente.

\section{Referências}

ALUR, D.; CRUPI, J.; MALKS, D.; Core J2EE Patterns: Best Practices and Design Strategies, Califórnia, Sun Microsystems Press, 2001.

DEITEL, H.M.; DEITEL, P.J.; Java: Como

Programar, 6 ed. São Paulo, Pearson, 2006.

DESHMUKH, H.; MALAVIA, J.; SCWCD, Exam Study Kit: Java Web component Developer Certification, Greenwich, 2003.
PAULA, A.M.; Gerenciamento, Análise

e Transmissão de imagens, On-Line, Via

TCP/IP, Dissertação de Mestrado, Mestrado em Tecnologia, CEFET, Rio de Janeiro, RJ, Brasil, 2007.

SDN Sun Developer Network: JavaServer Faces Technology, 2009. Disponível em:< http://java.sun.com/javaee/javaserverfaces/> Acesso em: 18 Mai. 2009.

STRUTS The Apache Software Foundation, 2009. Disponível em: <http://struts.apache. org> Acesso em: 18 Mai. 2009.

Endereço para Correspondência:

Angelo Márcio de Paula

Coordenação de Sistema de Informação

e Engenharia Elétrica

angelo.paula@foa.org.br

Centro Universitário de Volta Redonda

Campus Três Poços

Av. Paulo Erlei Alves Abrantes, $n^{\circ}$ 1325,

Três Poços - Volta Redonda / RJ

CEP: $27240-560$ 